\title{
ONE-DIMENSIONAL BASIC SETS IN THE THREE-SPHERE
}

\begin{abstract}
BY
JOEL C. GIBBONS

Abstract. This paper is a continuation of Williams' classification of one-dimensional attracting sets of a diffeomorphism on a compact manifold [Topology 6 (1967)]. After defining the knot presentation of a solenoid in $S^{3}$ and some knottheoretic preliminaries, we prove Theorem: If $\Sigma_{1}, h_{1}$ and $\Sigma_{2}, h_{2}$ are shift classes of oriented solenoids admitting elementary presentations $K, g_{1}$ and $K, g_{2}$, resp., where $g_{1}{ }^{*}=\left(g_{2}{ }^{*}: H_{1}(K) \rightarrow H_{1}(K)\right.$, there is an Anosov-Smale diffeomorphism $f$ of $S^{3}$ such that $\Omega(f)$ consists of a source $\Lambda^{-}$and a sink $\Lambda^{+}$for which $\Lambda^{+}, f / \Lambda^{+}$and $\Lambda^{-}, f^{-1} / \Lambda^{-}$ are conjugate, resp., to $\Sigma_{1}, h_{1}$ and $\Sigma_{2}, h_{2}$. (The author has proved [Proc. Amer. Math. Soc., to appear] that if $f$ is an Anosov-Smale map of $S^{3}, \Omega(f)$ has dimension one, and contains no hyperbolic sets, then $f$ has the above structure.) We also prove Theorem: there is a nonempty $C^{1}$-open set $F_{2}$ in the class of such diffeomorphisms for which $K=S^{1}$ and $g_{1}=g_{2}$ is the double covering such that each $f$ in $F_{2}$ defines a loop $t$ in $S^{3}$, stable up to $C^{1}$ perturbations, for which at every $x$ in $t$ the generalized stable and unstable manifolds through $x$ are tangent at $x$.
\end{abstract}

0. Introduction. The purpose of this paper is to construct a family of examples of Anosov-Smale diffeomorphisms of $S^{3}$. Following Smale's characterization of diffeomorphisms by labeled diagrams [1], the problems of realizing some possible diagrams arose. The one we are interested in consists of two basic sets-a source and a sink. In particular, we will construct the family of these diffeomorphisms for which the source and sink are 1-dimensional and oriented.

The methods we use are in essence drawn from [2]. Williams defines solenoid, $\Sigma, h$, and elementary presentation $K, g$ of a solenoid. $K$ is a smooth wedge of 1 spheres, and $g$ is an immersion of $K$. A transpose $g^{t}$ of $g$ is an immersion of $K$ such that $K, g^{t}$ is a presentation of a solenoid, $\Sigma^{t}, h^{t}$, and $g_{*}^{t}=\left(g^{t}\right)_{*}: H_{1}(K) \rightarrow H_{1}(K)$. After considerable knot-theoretic preliminaries, we prove

LEMMA 3.2. Every elementary presentation $K, g$ admits a transpose $K, g^{t}$, and

THEOREM 3.3 Let $\Sigma, h$ be a solenoid with elementary presentation $K, g$. Let $K, g^{t}$ be any transpose, generating $\Sigma^{t}, g^{t}$. There is an Anosov-Smale diffeomorphism $f$ of $S^{3}$ such that

(a) $\Omega(f)=\Lambda^{+} \cup \Lambda^{-}, \Lambda^{+}$a source, $\Lambda^{-}$a sink,

(b) $\Lambda^{+}, f^{-1} / \Lambda^{+}$is topologically conjugate to $\Sigma, h$ and $\Lambda^{-}, f / \Lambda^{-}$is topologically conjugate to $\Sigma^{t}, h^{t}$.

Presented to the Society, September 2, 1971; received by the editors November 16, 1970.

AMS 1970 subject classifications. Primary 34C 35, 54H20, 55A25, 58F15.

Key words and phrases. Generalized solenoid, structural instability, knot factorization.

Copyright (c) 1972, American Mathematical Society 
We note that no larger class of pairs of solenoids occur as source and sink of an Anosov-Smale diffeomorphism of $S^{3}$. We also prove that our diffeomorphisms are not structurally stable, and in particular

Proposition 4.2. There is a diffeomorphism of the type we have defined which has a loop of points of tangency of stable and unstable manifolds of $f$. This property is $C^{1}$ stable.

I would like to thank Professors Robert Williams, Daniel Kahn, and Mark Mahowald for their many helpful comments.

1. Topological preliminaries. Our basic procedure for constructing solenoids is derived from [2]. The first step is to define open sets $N$ and $M$ such that $S^{3}=N$ $\cup M \cup \delta N$ and $\delta N=\delta M=T$, and then defining a diffeomorphism $f$ such that $f(M \cup T) \subset M$.

DEFINITION 1.1. $K$ is an elementary branched 1-manifold if it is a wedge of onespheres.

Henceforth, we will be interested exclusively in smooth, oriented elementary branched 1-manifolds, which we call branched knots.

If $K$ is a branched knot and $i: K \rightarrow S^{3}$ is an embedding, $i$ is simple if there is a family of two-discs $D_{1}, \ldots, D_{k}$ such that each loop of $i(K)$ bounds a disc, and any two are disjoint. For simplicity, we will identify $K$ with $i(K)$ and refer to $K$ (as a subset of $S^{3}$ ) as a simple branched knot.

DEFINITION 1.2. $N_{n}$, for any positive integer $n$, is a fundamental neighborhood (of $K$ ) if (a) $N_{n}$ is an open subset of $S^{3}$, (b) $\delta N_{n}$ is a smooth two-manifold of genus $n$, and (c) $N_{n}$ contains the simple branched knot $K$ as a deformation retract. We will also use $M_{n}$ to refer to a fundamental neighborhood, and $L$ a simple branched knot. Where it does not enter the argument, we will suppress the subscript denoting the genus of $\delta N_{n}$.

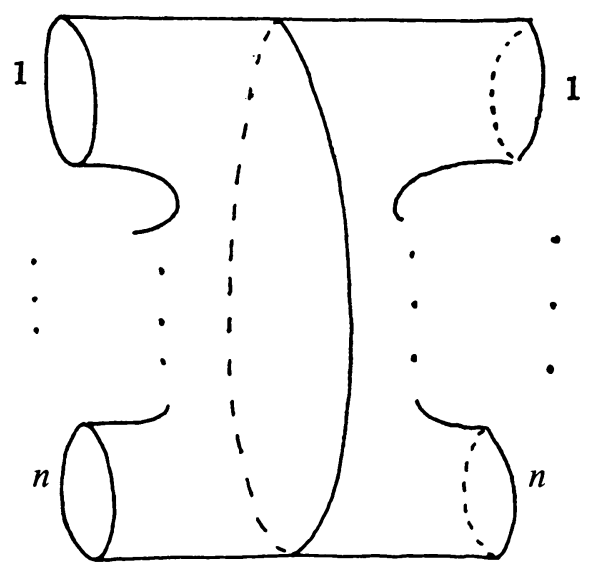

FIGURE 1 
$N_{n}$ is diffeomorphic to the quotient space obtained by attaching the "stubs" of the figure shown according to the numbers.

$N$ admits a decomposition into disjoint smoothly embedded open two-discs corresponding under the quotient map to the vertical slices of the figure above. We refer to this decomposition as the foliation $\mathscr{G} N$. Note that this foliation has the property that if $N$ is a fundamental neighborhood of $K$ we may choose the foliation so that $K$ is transversal to all of the leaves. In this case, we say $K$ is transversal to $\mathscr{G} N$.

Let $\delta N_{n}=T . H_{1}(T)$ admits a basis $\alpha_{1}, \ldots, \alpha_{n}, \beta_{1}, \ldots, \beta_{n}$ defined as follows: Let $K=\bigvee^{n} k_{j}$; if $G_{j}$ is any leaf of $\mathscr{G}_{N}$ such that $G_{j} \cap K \subset k_{j}, \delta G_{j}$ has homology $\alpha_{j}$, oriented so that $\delta G_{j}$ has linking +1 with $k_{j}$. $\beta_{j}$ contains a loop homologous in $N_{n}$ to $k_{j}$. Then the leaves of $\mathscr{G} N$ are of two types, those with boundary homologous to a generator of $H_{1}(T)$ and those with homology $\alpha_{1}+\alpha_{2}+\cdots+\alpha_{n}$. The union of all the leaves of the latter type is called the body of $N_{n}, \mathscr{B} N$.

Having defined one of our desirable neighborhoods, we want next to "fill up" $S^{3}$ with two such.

Proposition 1.3. If $N$ is a fundamental neighborhood of $K$ and $\delta N=T, S^{3}-T \cup N$ is a fundamental neighborhood. There is a diffeomorphism $\left(C^{\infty}\right)$ of $S^{3}$ such that if $M=S^{3}-T \cup N$

(a) $h(T)=T, h(N)=M$,

(b) $M$ is a fundamental neighborhood of $h(K)=L$,

(c) $h^{2}=$ identity of $S^{3}$, and

(d) $h$ carries $\mathscr{G} N$ to a foliation $\mathscr{G} M$ of $M$ transversal to $L$.

Proof. It suffices to construct $h$. Also, properties (b), (c), and (d) will follow from (a) by standard topological arguments. For simplicity, we refer to the points in $S^{3}$ by their images under some fixed stereographic projection. We assume that $N$ consists of the unit ball with handles which intersect the $x-y$ plane on discs. Suppose that $N$ has $n$ handles, and let $N^{*}$ consist of the unit ball with $n$ holes drilled in it. $N^{*}$ is isotopic to $N$; let $g$ be the map of $S^{3}$ which sends $N$ to $N^{*}$ defined by the isotopy. Let $h^{*}$ be the diffeomorphism, in polar coordinates, $h^{*}(r, \theta, \phi)=(1 / r, \theta, \phi)$. The diffeomorphism $h$ is completed by an isotopy which sends $h^{*} \cdot g(N)$ to $M$, i.e. $h$ is the composite function. Q.E.D.

This diffeomorphism is merely a realization of the isomorphism of $H^{1}(\bar{N})$ to $H_{1}(M)$ defined by Alexander duality (up to some natural identification). This completes the topological groundwork.

2. Knot presentation of a solenoid. Let $\Sigma, h$ be a solenoid with smooth, oriented elementary presentation $K, g$. Let $K=\bigvee_{j}^{n} k_{j}$, and let $\kappa_{j}$ be the homotopy class of $k_{j}$ rel. the branch point. $\Pi_{1}(K, p)$ is the free nonabelian group on the $n$ letters $\kappa_{1}, \ldots, \kappa_{n}$; identify $\kappa_{j}$ with its image under the quotient map $\Pi_{1}(K, p) \rightarrow H_{1}(K)$. By abuse of language we will identify $g$ with its induced homomorphism on homotopy, because the homotopy lift identifies $g$ up to isotopies of $K$ which leave $p$ fixed. Let 
$g_{*}$ be the induced map on homology. Since $g$ is a surjective immersion, $g_{*}$, expressed in the basis $\kappa_{j}$, is an $n \times n$ integer matrix with no negative entries and no row or column of zeros. Conversely, given such a matrix $A$ we can find a $g$ such that $A=g_{*}$, so we begin by defining a knot presentation of a matrix $A$.

Definition 2.1. Let $A$ be an $n \times n$, nonnegative integer matrix and let $N_{n}$ and $M_{n}$ be fundamental neighborhoods of $K_{0}$ and $L_{0}$ respectively as in Proposition 1.3. Orient the knots so the $j$ th loop of $K_{0}, k_{j}^{0}$, links the $i$ th loop of $L_{0}, l_{i}^{0}$, with linking number $\delta_{j i}$. In general, if $K$ and $L$ are simple branched knots with $n$ loops each, $l(K, L)$ is the $n \times n$ matrix whose jith entry is the linking number of the $j$ th loop of $K$ with the $i$ th loop of $L$.

Now, choose simple branched knots $K_{1}$ and $L_{1}$ satisfying

(a) $K_{1} \subset N_{n}, L_{1} \subset M_{n}$,

(b) $K_{1}$ is transversal to $\mathscr{G} N, L_{1}$ is transversal to $\mathscr{G} M$,

(c) $l\left(K_{1}, L_{0}\right)=A, l\left(L_{1}, K_{0}\right)=A^{t}$, and

(d) there are isotopies $\phi$ and $\phi^{\prime}$ of $S^{3}$ such that $\phi\left(1, K_{1}\right)=K_{0}$ and $\phi^{\prime}\left(1, L_{1}\right)=L_{0}$. Then, the six-tuple $\left(K_{1}, L_{0}, N, M, \mathscr{G} N, \mathscr{G} M\right)$ is a knot presentation of $A$. The reader may satisfy himself that, if $A$ has no row or column of zeros, $A$ and $A^{t}$ admit knot presentations. (Example: Figure 2.) Where no confusion results we will refer to the knot presentation simply as $\left(K_{1}, L_{0}\right)$ since these determine the other elements up to isotopies of $S^{3}$.

Definition 2.2. A knot transposition of $\left(K_{1}, L_{0}, N, M, \mathscr{G} N, \mathscr{G} M\right)$ is an isotopy $\phi$ of $S^{3}$ such that $\left(\phi\left(1, L_{0}\right), K_{0}, M, N, \mathscr{G} M, \mathscr{G} N\right)$ is a knot presentation of $A^{t}$.

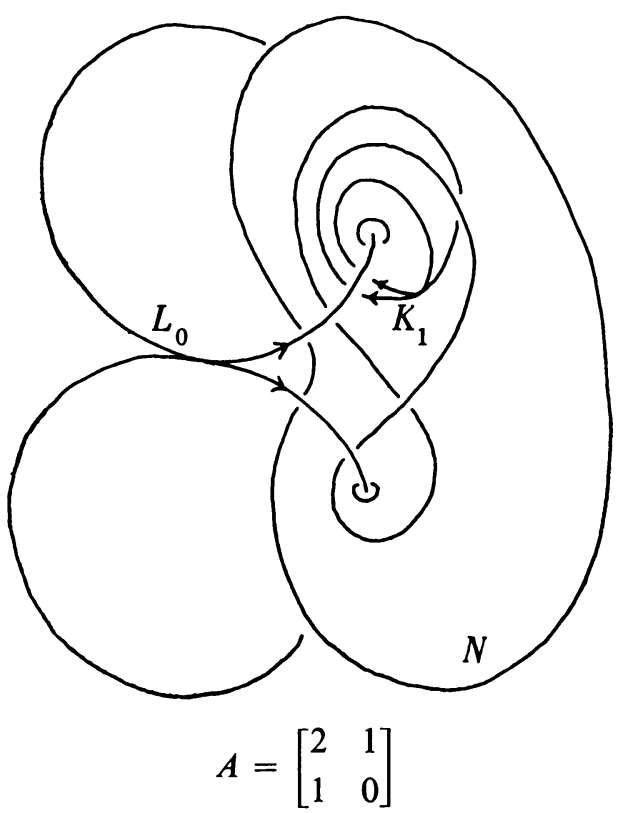

Figure 2 
Having straightened out the notation, we are ready to particularize the concepts of knot presentation and knot transposition to surjective immersions $g$ of $K$. As before, identify $g$ with its induced map on homotopy; suppose that $g\left(\kappa_{j}\right)=w_{j}$, $j=1, \ldots, n$. Since $g$ is an immersion no $w_{j}$ is trivial, and only positive powers of the generators occur; $w_{j}$ is called a strictly positive word, $g_{*}$ is a matrix as above; a knot presentation of $g$ is a knot presentation of $g_{*}$ with the additional property

(e) identify $\Pi_{1}(K, p)$ with $\Pi_{1}(N, p)$ via the embedding of $K_{0}$ into $N$, and so represent $\Pi_{1}(N, p)$ as the free group on the letters $\kappa_{j}, j=1, \ldots, n$. Then, if $k_{j}^{1}$ is the $j$ th loop of $K_{1},\left[k_{j}^{1}\right]=w_{j}$.

EXAMPLE. The knot presentation of the preceding example is $g\left(\kappa_{1}\right)=\kappa_{1} \kappa_{2} \kappa_{1}$, $g\left(\kappa_{2}\right)=\kappa_{1}$. If $\left(K_{1}, L_{0}, N, M, \mathscr{G} N, \mathscr{G} M\right)$ is a knot presentation of $g$, a knot transposition is a knot transposition of $g_{*}$.

Notation. If $U$ is an open subset of $S^{3}$, a perturbation of $U$ is an isotopy of $S^{3}$ which is constant on the complement of $U$.

TheOREM 2.3. Let $g: K \rightarrow K$ be a surjective immersion of $K$ with knot presentation $\left(K_{1}, L_{0}, N, M, \mathscr{G} N, \mathscr{G} M\right)$. Let $\phi$ be an isotopy of $S^{3}$ such that $\phi\left(1, K_{1}\right)=K_{0}$ and $\phi\left(1, L_{0}\right)$ is contained in $M$. Then there is a perturbation $\psi$ of $M$ such that $\psi * \phi$ is a $k n o t$ transposition of $g$ if and only if there is a homotopy of $M$ which carries $\phi\left(1, L_{0}\right)$ to a simple branched knot $L_{1}^{\prime}$ transversal to $\mathscr{G} M$.

To clarify the assertion we have made, we include some examples of (onelooped) branched knots satisfying the homotopy but not the isotopy condition. (Example: Figure 3.)
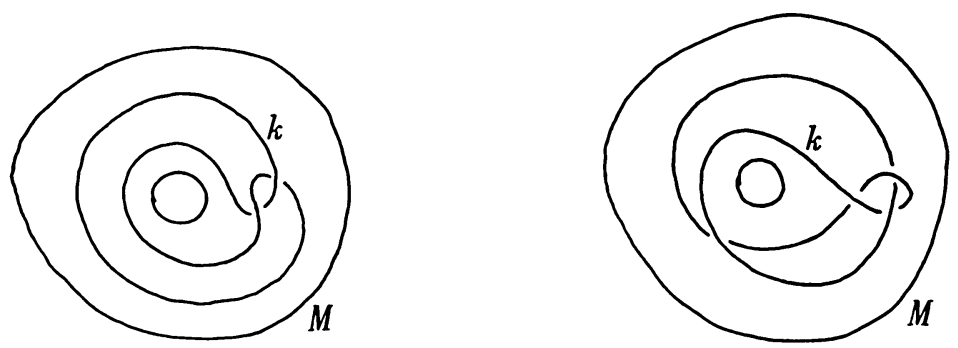

FIGURE 3

Proof. The proof of this proposition is rather involved, and depends on a sequence of lemmas about knots. What we will prove about knots concerns a relation on knots called factorization.

Definition 2.4. Let $U$ be an open set in $S^{3}$ and let $k, k_{1}$ and $k_{2}$ be embedded loops in $U$. $k$ factors to $\left\{k_{1}, k_{2}\right\}$ if there are distinct points $x, x^{\prime}, y$, and $y^{\prime}$ on $k$, a closed, contractible set $B$ such that $B \cap k$ consists of the arcs $\left[x, y^{\prime}\right]$ and $\left[x^{\prime}, y\right]$, and arcs $a$ and $a^{\prime}$ in $B$, from $x$ to $y$ and from $x^{\prime}$ to $y^{\prime}$, respectively, called crossing arcs, such that $\left\{a \cup[y, x], a^{\prime} \cup\left[y^{\prime}, x^{\prime}\right]\right\}$ is perturbable to $\left\{k_{1}, k_{2}\right\}$. (Example: Figure 4.) The loops $k_{1}$ and $k_{2}$ are called factors of $k$, of course, and as usual a factor of either 

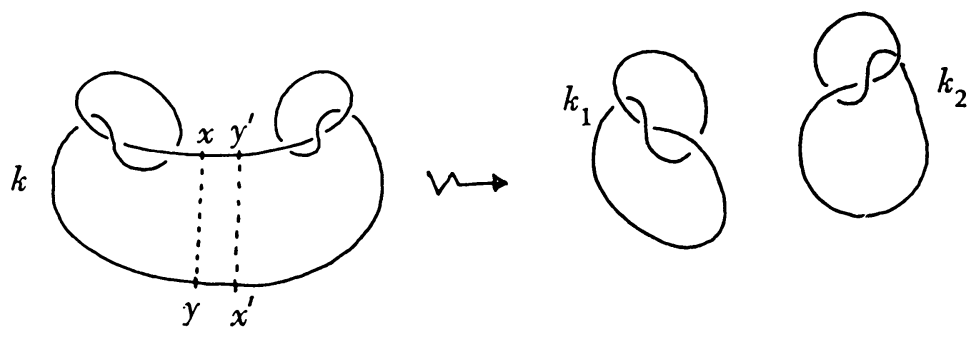

Figure 4

is a factor of $k$. This defines a transitive relation on the family of sets of embedded loops in $U$, where a factorization of a set of loops is the result of a sequence of factorizations of the member loops. In the case that $U=S^{3}$ and $k_{1}$ and $k_{2}$ lie on opposite sides of a two-sphere, this is factorization in the usual sense [3]; lacking some equivalent of the latter condition, factorization is in no way unique, but we will always be able to ignore irrelevant factorizations.

Note that we may assume without loss of generality that at every point at which a loop $k$ is tangent to a leaf $G$ of $\mathscr{G} N(\mathscr{G} M)$, the tangency is nondegenerate in the sense that a neighborhood in $k$ of the point lies entirely to one side of the leaf $G$.

LEMMA 2.5. Let $k$ and $k^{\prime}$ be embedded loops in $M$ such that

(a) there is a free homotopy of $M$ carrying $k$ to $k^{\prime}$,

(b) $k^{\prime} \pitchfork \mathscr{G} M$,

(c) $k^{\prime}$ has no negative linkings with loops of $K_{0}$, and

(d) $k$ has at most a finite number of tangencies to leaves of $\mathscr{G} M$.

Then, $k$ has a factorization in $M$ to $\left\{k_{1}, \ldots, k_{s}\right\}$ such that

(a*) $k_{1}$ 历 $\mathscr{G} M$,

(b*) there is a homotopy of $M$ carrying $k_{1}$ to $k^{\prime}$, and

(c*) $k_{j}, j=2, \ldots, s$, is freely homotopic to a point.

The converse is also true.

Proof. If $k_{1}, \ldots, k_{s}$ is any factorization of $k$, let $\bar{k}_{j}$ be the arc of $k$ contained in the factor $k_{j}$. By the transitivity of factorization, it suffices to define a method for factoring $k_{2}$ off of $k$, and to check that the inductive limit satisfies the conditions given. We may assume that every point of tangency lies in the body of $M$. By condition (a), $k$ is a strictly positive word in the homotopy group of $M$ at some point in the body. We need a way of calculating the homotopy of $k$ which gives in some sense a nonreduced word.

For this purpose, choose leaves $H_{j}, j=1, \ldots, n$, of $\mathscr{G} M$ where $H_{j}$ lies on the $j$ th handle of $M$, and a leaf $G_{2}$ contained in the body. Orient intersection with these discs by stipulating that $L_{0}$ intersects each in the positive direction. Perturb $k$ so that it intersects $G_{2}$ at least once between successive intersections with the $H_{j}$, and let $k \cap G_{2}=\left\{z_{1}, z_{2}, \ldots, z_{r}\right\}$. If the arc $\left[z_{i}, z_{i+1}\right]$ intersects $H_{j}$ in the positive (negative) sense, the $i$ th letter of $k$ is $h_{j}^{+1}\left(h_{j}^{-1}\right)$. If $\left[z_{i}, z_{i+1}\right]$ meets no $H_{j}$, the $i$ th letter is 
$h_{0}$. The reader may check to see that in this basis the reduced word for $[k]$ is strictly positive.

Let $x_{2}$ be a point at which $k$ is tangent to a leaf of $\mathscr{G} M$, and suppose that $G_{2}$ is this leaf. Let $v_{2}$ be the maximal trivial subword of the unreduced word for $[k]$ defined above containing a letter which represents an arc which has $x_{2}$ as an endpoint. Such a word must exist, because either the arc preceding $x_{2}$ or the arc following it is $h_{0}$ or one of these is a negative power of a generator.

Tentatively, let $\bar{k}_{2}^{\prime}$ be the arc of $k$ represented by $v_{2}$. Now, choose points $x$ and $y$ on $\bar{k}_{2}^{\prime}$ and $x^{\prime}$ and $y^{\prime}$ on $k-\bar{k}_{2}^{\prime}$ close to the endpoints. Choose crossing arcs $a_{2}$ and $a_{2}^{\prime}$ as shown, and let $k_{2}$ be the homotopically trivial loop (i.e. containing almost all of $\bar{k}_{2}^{\prime}$ ). The figure illustrates the two distinct cases, depending on whether $x_{2}$ is an endpoint of $\bar{k}_{2}^{\prime}$ or not. Now, the whole factorization is obtained by successively factoring the homotopically nontrivial factor until all the tangencies have been factored off (Figure 5). To see that this is possible, note that (1) $k$ has at most a
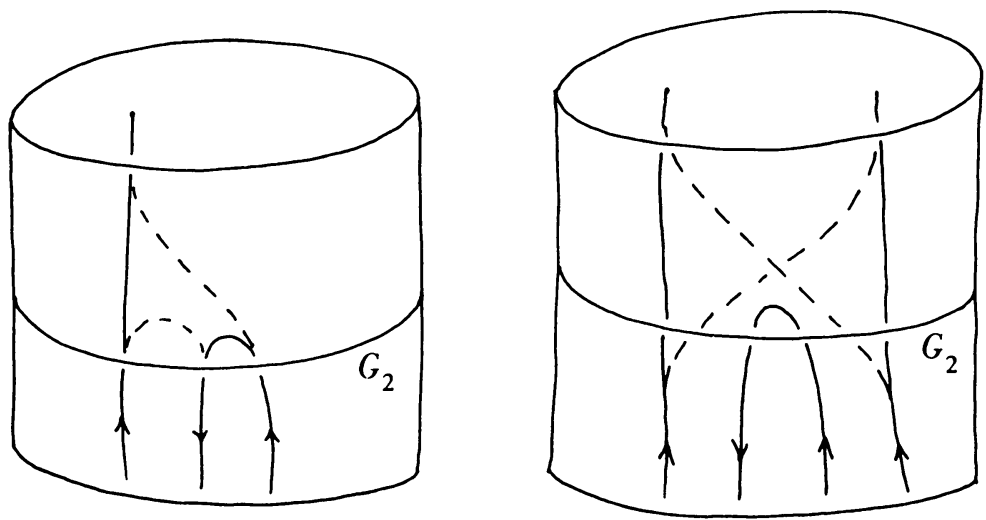

FIGURE 5

finite number of tangencies and (2) if the crossing arcs are joined smoothly to $k$ the factors have between them the same number of tangencies as the original loop, and (3) each trivial factor contains at least one tangency. We include an example of factorization in the large (Figure 6). The converse to this lemma is trivially true, since the homotopies of the factors $k_{2}, \ldots, k_{s}$ define a homotopy of $k$ to $k^{\prime}$. Q.E.D.

Now, we want to improve our choice of crossing arcs, under the right assumptions.

LEMMA 2.6. Let $k$ and $k^{\prime}$ be as above. There is a perturbation of $M$ carrying $k$ to $k^{\prime}$ if and only if $k$ has a factorization $\left\{k_{1}, \ldots, k_{s}\right\}$ such that $k_{1}$ is perturbable to $k^{\prime}$ and each $k_{j}, j=2, \ldots, s$, bounds an embedded disc in $M-\bigcup_{i \neq j} k_{i}$.

Proof. Sufficiency of the disc bounding property is obvious; the isotopy is taken to contract the discs. To prove necessity we will have to be more explicit about the choice of crossing arcs. 
Let $\phi$ be the isotopy; $\phi(1, k)=k^{\prime}$. As before, we will prove the proposition in detail for $k_{2}$ only. Let $\phi\left(1, k_{2}^{\prime}\right)=c_{2}$, and $\phi\left(1, G_{2}\right)=G_{2}^{\prime}$. $G_{2}^{\prime}$ is not necessarily contained in the body of $M$, but by "sliding it along" $k$ ' we may translate it into the body. At this stage we have the following picture (Figure 7). We want to find an
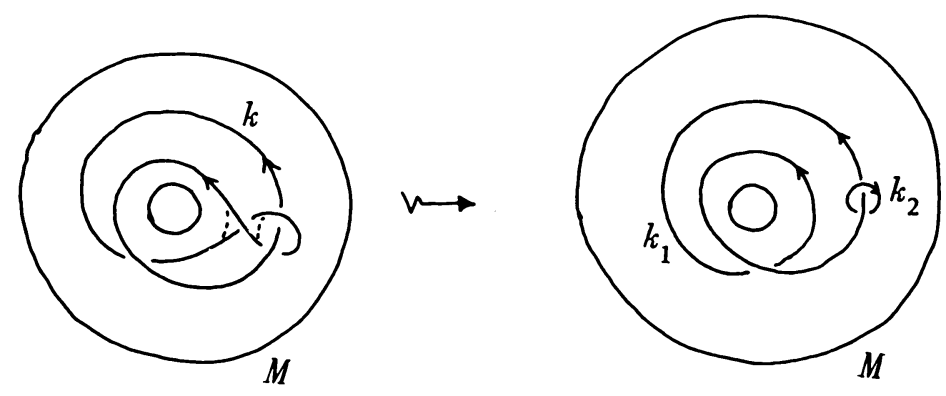

FIGURE 6

arc $w^{\prime}$ in $\mathscr{B} M$, smoothly joined to $c_{2}$ at the endpoints, lying in an arbitrarily small neighborhood of $G_{2}^{\prime}$ such that $w^{\prime} \cup c_{2}$ bounds a disc in $M-\left(k^{\prime}-c_{2}\right)$. To see that such an arc exists, note that there is an isotopy of $M$ which carries $c_{2}$ to such an arc.

Let $w$ be the arc in $M$ such that $\phi(1, w)=w^{\prime}$. At this stage we have the following figure (Figure 7). If $\bar{k}_{2}^{\prime}$ intersects $G_{2}$ between its endpoints, apply this procedure

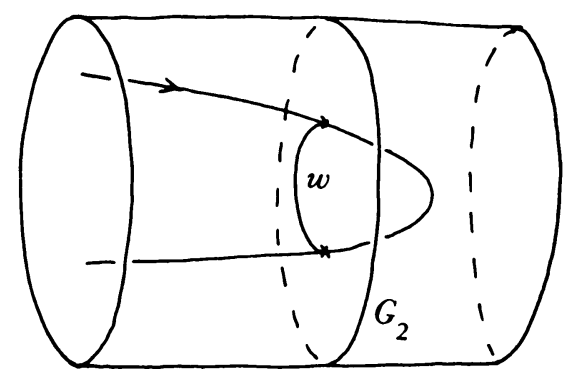

FIGURE 7

successively to the subarcs of $\bar{k}_{2}^{\prime}$ disjoint from $G_{2}$ with endpoints in $G_{2}$. For crossings arcs choose any sufficiently close approximations to $w$.

The result stated follows from the successive application of this procedure to the $s-1 \operatorname{arcs} k_{j}^{\prime}, j=2, \ldots, s$. Q.E.D.

Now, we must justify the hypotheses of these lemmas and apply the results to branched knots.

LEMMA 2.7. Let $\phi$ be the isotopy defined in the statement of the theorem. If $\phi$ is $C^{1}$, $L_{1}=\phi\left(1, L_{0}\right)$ has at most a finite number of points of tangency to leaves of $\mathscr{G} M$.

Proof. If $\phi$ is a $C^{1}$ isotopy, the embedding of $L_{1}$ is a $C^{1}$ immersion (if $L_{0}$ is $C^{1}$ ). Hence, we need only show that if $L_{1}$ has an infinite number of tangencies, it is not an immersion. 
Suppose that $L_{1}$ has an infinite number of points of tangency and let $a$ be the limit of the sequence $\left\{a_{k}\right\}$ of such points. Take a neighborhood of $a$ in $M$ and give it cylindrical coordinates $r, \theta, z$ so that the $z$ coordinate is constant on leaves of $\mathscr{G} M$. Choose balls $B_{k}$ in $M$ such that $B_{k} \cap L_{1}$ is an arc lying between $a_{k}$ and $a_{k+1}$. Perturb $L_{1}$ to an embedding $L_{1}^{\prime}$ which is equal to $L_{1}$ outside the balls $B_{k}$ and in each one there is a point at which $L_{1}^{\prime}$ is tangent to the $z$-axis. It suffices to show that $L_{1}^{\prime}$ is not an immersion. If $L_{1}^{\prime}\left(t_{k}\right)=a_{k}$ and $L_{1}^{\prime}\left(s_{k}\right)$ is a point in $B_{k}$ at which $L_{1}^{\prime}$ is parallel to the $z$-axis, and $L_{1}^{\prime}(t)=a$,

$D\left(z \cdot L_{1}^{\prime}\right)(t)=\lim _{k \rightarrow \infty} D\left(z \cdot L_{1}^{\prime}\right)\left(t_{k}\right)=0$,

$D\left(r \cdot L_{1}^{\prime}\right)(t)=\lim _{k \rightarrow \infty} D\left(r \cdot L_{1}^{\prime}\right)\left(s_{k}\right)=0$, and

$D\left(\theta \cdot L_{1}^{\prime}\right)(t)=\lim _{k \rightarrow \infty} D\left(\theta \cdot L_{1}^{\prime}\right)\left(s_{k}\right)=0$. Q.E.D.

Thus, in the following we need not assume that $L_{1}$ has at most a finite number of tangencies, though we will need that fact.

Definition 2.8. If $M$ is a fundamental neighborhood and $L$ is a simple branched knot in $M$, a factorization of $L$ is a factorization of one or more loops of $K$.

LEMmA 2.9. Let $K_{1}, K_{0}, L_{1}, L_{0}$, and $\phi$ be defined as in the statement of the theorem. Let $L_{1}^{\prime}$ be a simple branched knot in $M$ transversal to $\mathscr{G} M$. There is a free homotopy of $M$ carrying $L_{1}$ to $L_{1}^{\prime}$ if and only if there is a factorization $\left\{L, k_{2}, \ldots, k_{s}\right\}$ of $L_{1}$, where $L$ is a simple branched knot transversal to $\mathscr{G} M$ and ambient homotopic to $L_{1}^{\prime}$ and the loops $k_{2}, \ldots, k_{s}$ are ambient homotopic to points. Further, $L_{1}$ is perturbable to $L_{1}^{\prime}$ if and only if each loop $k_{j}$ bounds a disc in $M-L \cup\left(\cup_{j \neq i} k_{i}\right)$.

Proof. The second conclusion follows directly from the existence of the factorization and the methods of Lemma 2.6. In proving the first assertion, the methods of Lemma 2.5 apply without change except we must insure that no trivial factor contains the branch point.

Define arcs of $L_{1}$ by the factorization, as before, and represent the homotopy group at the branch point of $L_{1}$ as before (Lemma 2.5). From the homotopy condition, we may assume that each loop of $L_{1}$ represents in this group a strictly positive word. We may also assume that a point of tangency $x_{2}$ and the branch point $q$ lie in the same leaf of $\mathscr{G} M, G_{2}$.

Suppose $x_{2}$ is in $k_{1}^{1}$, the first loop of $L_{1}$, and let $v_{2}$ be the maximal trivial subword of $\left[k_{1}^{1}\right]$ containing a letter representing an arc beginning or ending with $x_{2}$, and call the arc represented by $v_{2} k_{2}^{*}$. If $q$ occurs in the interior of $k_{2}^{*}$ it occurs at the start of a letter of $v_{2}$ and the end of the predecessor. Factor $v_{2}$ to $v_{2}^{\prime} \cdot v_{2}^{\prime \prime}$, where $v_{2}^{\prime}$ ends and $v_{2}^{\prime \prime}$ begins with $q$. Then $\left[k_{1}^{1}\right]=v_{2}^{\prime \prime} \cdot v \cdot v_{2}^{\prime}$, but since $v_{2}$ is maximal, this implies that $\left[k_{1}^{1}\right]$ is not strictly positive. Q.E.D.

This completes our characterization of transversality up to ambient homotopy and isotopy; the general conclusion is that if $L_{1}$ is perturbable to a transversal simple branched knot, the perturbation can be taken to be constant outside a contractible set. The proof of the theorem is completed by using the isotopy $\phi$ and the duality map $h$ to compare the embeddings $K_{1}$ and $L_{1}$. 
LEMMA 2.10. Let $U$ be the interior of $\mathscr{B} M$ and $V$ be the interior of $\mathscr{B} N$. All other terms as defined above. There is a diffeomorphism $F: U \rightarrow V$ such that

$$
F\left(U \cap L_{1}\right)=V \cap K_{1}
$$

Proof. Let $\phi^{-1}$ be the isotopy inverse to $\phi$, and let $\phi_{1}^{-1}$ be the diffeomorphism defined by $\phi^{-1}$ at 1 . Let $U_{1}=\phi_{1}^{-1}(U)$. By perturbing $\phi$ if necessary we may arrange that $U_{1} \cap K_{0}=\varnothing ; U_{2}=h\left(U_{1}\right)$. We may therefore perturb $\phi$ so that $\phi_{1}^{-1}\left(U_{2}\right) \subset N$; and necessarily $\phi_{1}^{-1} \cdot h \cdot \phi_{1}^{-1}\left(U \cap L_{1}\right)$ is contained in $K_{1}$. Let this composite function be $g$. It only remains to perturb $g$ to a diffeomorphism which maps $U$ onto $V$. This can be done if there is no component of $K_{1} \cap V$ which does not meet the image of $g$, and that is clearly the case because $K_{1} \cap V$ has no more components than $L_{1} \cap U$. Q.E.D.

We note that in general this diffeomorphism does not extend to $M$, since the linking matrices $l\left(K_{1}, L_{0}\right)$ and $l\left(L_{1}, K_{0}\right)$ are usually not equal.

Clearly $F$ is defined only up to perturbations of $N$, so in applying the lemma we will feel free to modify $F$ by perturbations whenever desirable.

The application of these lemmas is now rather apparent.

If $L_{1}$ has a point of tangency to a leaf of $\mathscr{G} M$, assume it is in $\mathscr{B} M$, and let $\left\{L, k_{2}\right\}$ be any factorization of $L_{1}$ defined by the tangency. Since the crossing arcs are contained in $\mathscr{B} M$, this factorization defines via $F$ a factorization of $K_{1}$. Now, we may choose crossing arcs for this factorization so that the homotopically trivial loop bounds a disc, and we may perturb $F$ so that its image contains this disc. This disc defines via $F^{-1}$ an isotopically trivial factorization of $L_{1}$ at $x_{2}$. Now, apply this argument inductively to the tangencies of $L_{1}$.

On the basis of these knot techniques we will prove in the next section that every surjective immersion $g$ of an oriented, elementary branched 1-manifold admits a knot transposable knot presentation.

3. Construction of attracting sets. To apply the theorem of the preceding section to the problem of transposing maps, we must translate its conclusion into an initial condition on knot presentations. This is done quite easily as follows.

With all symbols as defined before, let $\mathscr{D}$ be a family of disjoint discs $D_{j}, j=1, \ldots$, $n$, such that $\delta D_{j}=k_{j}^{1}$, the $j$ th loop of $K_{1}$. Since $\left(K_{1}, L_{0}\right)$ is a knot presentation of some map, there is an isotopy $\phi$ of $S^{3}$ such that $\phi\left(1, K_{1}\right)=K_{0}$; let $C_{j}=\phi\left(1, D_{j}\right)$. Orient intersection of arcs with these discs by the orientation of $K_{1}$ and the righthand rule (i.e. a loop which intersects $D_{j}$ once in the positive sense has linking number +1 with $k_{j}^{1}$ ). Lastly, let $C_{j} \cap M=C_{j}^{\prime}$.

Proposition 3.1. If there is a foliation $\mathscr{G} M$, as defined above, such that the $C_{j}^{\prime}$ are leaves and if each loop of $L_{0}$, wherever it intersects each disc $D_{j}$, intersects in the positive sense, there is a perturbation $\psi$ of $M$ such that $\psi * \phi$ is a knot transposition of $\left(K_{1}, L_{0}, N, M, \mathscr{G} N, \mathscr{G} M\right)$. 
Proof. To relate the terminology to that used in the proof of Theorem 2.3, let $C_{j}^{\prime}=H_{j}$. Then the conclusion follows from the fact that the isotopy preserves signed intersections and Theorem 2.3.

For clarity, we include an example of a knot presentation which admits no knot transposition. The first picture shows that our example is a knot presentation, the following ones how an isotopy fails irrevocably to be a knot transposition (Figure 8). This example notwithstanding, we will prove that every surjective
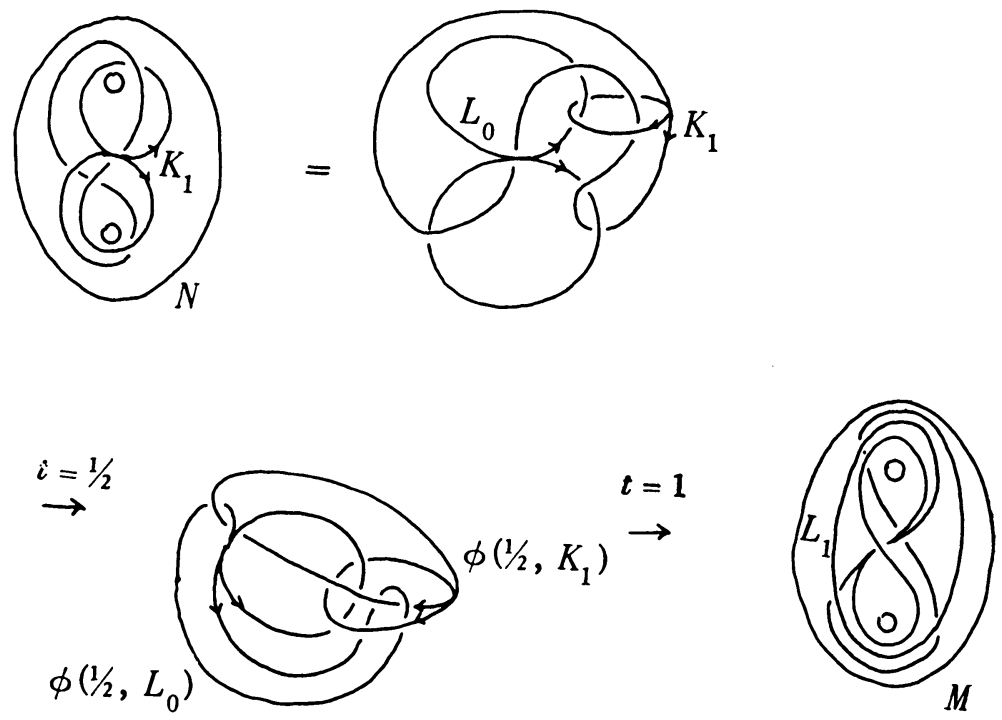

FIGURE 8

immersion $g$ of an elementary branched 1-manifold $K$ admits a knot transposable knot presentation. Before we state this result formally, we need a definition.

Definition 3.2. If $g$ is any such map, $g^{t}$ is a transpose if $g, g^{t}: K \rightarrow K$ are smooth maps and $g_{*}^{t}=\left(g^{t}\right)_{*}: H_{1}(K) \rightarrow H_{1}(K)$. As before, identify $g$ with its induced map on homotopy, and suppose that $g\left(\kappa_{j}\right)=w_{j}, g^{t}\left(\kappa_{j}\right)=w_{j}^{t}$.

Proposition 3.3. Let $g$ be any surjective immersion of $K$ and let $g^{t}$ be any transpose. There is a knot presentation $\left(K_{1}, L_{0}\right)$ of $g$ which admits a knot transposition $\phi$ to a knot presentation $\left(L_{1}, K_{0}\right)$ of $g^{t}$.

Proof. The proof is highly pictorial, so only an outline sufficient to guide the reader will be given. Let $w_{j}^{t}=\kappa_{j 1} \kappa_{j 2} \cdots \kappa_{j m_{j}}, j=1, \ldots, n$, and let $m=m_{1}+\cdots+m_{n}$. Choose $m$ disjoint loops centered at points on the unit circle in the $x-y$ plane and label them $c_{11}, \ldots, c_{1 m_{1}}, c_{21}, \ldots, c_{n m_{n}}$. Embed a loop in the ball of radius two which links these loops in the following way: suppose the first letter of $w_{1}$ is $\kappa_{j}$, and suppose that $\kappa_{1}$ is the $r$ th letter of $w_{j}^{t}$. Then the loop crosses the $x-y$ plane at the origin and next crosses the $x-y$ plane at the center of $c_{j r}$. If the second letter of $w_{1}$ is $\kappa_{k}$, and $\kappa_{1}$ is the sth letter of $w_{k}^{t}$, the loop next crosses the $x-y$ plane at the center of 
$c_{k s}$. Continue until each circle is linked once. Let $c_{j}, j=1, \ldots, n$, be a loop in the $x-y$ plane which contains the loops $c_{j q}, q=1, \ldots, m_{j}$, in its interior and all others in its exterior, and crosses the sphere of radius two.

Then $N$ is a smoothing of the ball of radius two minus tubes around the loops $c_{j}$. $K_{1}$ is formed by attaching $n$ points on the loop defined. The details of the construction are left to the reader. Q.E.D.

If $\phi$ is a knot transposition of $\left(K_{1}, L_{0}, N, M, \mathscr{G} N, \mathscr{G} M\right)$, let $f$ be the diffeomorphism $\phi_{1}$, where $\phi_{1}(x)=\phi(1, x)$. In general $f$ is not an Anosov-Smale diffeomorphism. In particular, if $\left(K_{1}, L_{0}\right)$ is a knot presentation of the identity, $f$ cannot be Anosov-Smale. However, the situation is different if $\left(K_{1}, L_{0}\right)$ and $\left(L_{1}, K_{0}\right)$ are presentations of maps satisfying Williams' solenoidal axioms [2]. Before we state the appropriate result, we need a lemma.

LEMMA 3.4. Let $g: K \rightarrow K$ be an immersion satisfying the solenoidal axioms. $g$ has a transpose which does also.

Proof. As Williams shows [2], there is an integer $m$ such that all entries of $g_{*}^{m}$ are positive. Then, all entries of $\left(g^{t}\right)_{*}^{m}$ are also, so if $g^{t}$ is any transpose, $\left(g^{t}\right)^{m}$ maps each loop of $K$ onto $K$. We take the axioms in order.

(a) Indecomposability will follow from proving that $\operatorname{Per}\left(g^{t}\right)$ is dense in $K$, because this will imply that, for each $\varepsilon>0$, there is a closed orbit which is an $\varepsilon$-net.

(b) From the fact that some iterate of $g^{t}$ maps each loop of $K$ onto $K$, we conclude that $g^{t}$ is expanding.

(c) That $\Omega\left(g^{t}\right)=K$ follows directly from (b) and the fact that some iterate of $g$ maps each loop onto $K$. We might note that so far we have hardly limited the choice of acceptable $g^{t}$, except to rule out local peculiarities in (a). The last axiom is the only one which narrows the field significantly.

(d) We must show that there is a transpose of which some power maps a neighborhood of the branch point to an interval, i.e. some power starts each loop off and ends each with the same loops. That this is true follows from the fact that if $g$ satisfies axiom (d), some $w_{j}$ starts with the letter $\kappa_{j}$. This is a simple consequence of axiom (d). Then if $K$ has $n$ loops, it is easy to choose a transpose for which $\left(g^{t}\right)^{n}$ starts each word off with $\kappa_{j}$. A similar argument for the last letters yields the conclusion.

We are at last ready for the main result.

THEOREM 3.5. Let $\Sigma, h$ be any solenoid admitting an elementary, oriented presentation $K, g$, and let $K, g^{t}$ be any transpose of $K, g$ which generates a solenoid $\Sigma^{t}, h^{t}$. There is an Anosov-Smale diffeomorphism $f$ of $S^{3}$ such that $\Omega(f)$ is the union of two basic sets $\Lambda^{+}$and $\Lambda^{-}$, and

(a) $\Lambda^{-}\left(\Lambda^{+}\right)$is a one-dimensional sink of $f\left(f^{-1}\right)$,

(b) $\Lambda^{+}, f^{-1} / \Lambda^{+}$is topologically conjugate to $\Sigma, h$, and $\Lambda^{-}, f / \Lambda^{-}$is topologically conjugate to $\Sigma^{t}, h^{t}$. 
Proof. Choose a knot presentation of $g$ which admits a knot transposition to a knot presentation of $g^{t}$, and let $f$ be the diffeomorphism induced from the transposition, as above. Let $\Lambda^{+}=\bigcap_{k=1}^{\infty} f^{-k}(N)$ and $\Lambda^{-}=\bigcap_{k=1}^{\infty} f^{k}(M)$. We must check a host of properties of $f$ :

Claim 1. $\Omega(f) \subset \Lambda^{+} \cup \Lambda^{-}$. Let $x \in S^{3}-\Lambda^{+} \cup \Lambda^{-}$, and suppose that $x \in f^{k}(M)$ $-f^{k+1}(M)$, for some integer (positive or negative) $k$. Then, there is a ball $U$ about $x$ contained in $f^{k}(M)-f^{k+1}(M)$. For any positive integer $m, f^{m}\left(f^{k}(M)-f^{k+1}(M)\right)$ $\subset f^{k+1}(M)$, so $x$ is not nonwandering.

Claim 2. $\Lambda^{+}, f^{-1} / \Lambda^{+}$is a solenoid topologically conjugate to $\Sigma, h . f^{-1}$ maps $N$ into itself and preserves a foliation $\mathscr{G} N$ transversal to $K_{1}$. Then $N / \mathscr{G} N$ is a smooth (not elementary) branched 1-manifold $K^{*}$, and $f^{-1}$ induces an immersion $g^{*}$ of $K^{*}$ such that the following commutes:

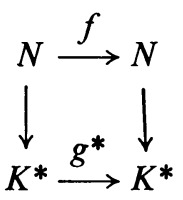

$K^{*}$ may be viewed as $K$ with a neighborhood (in $K$ ) of the branch point smoothly collapsed. Since some iterate of $g$ collapses this neighborhood to a line segment, $K, g$ is shift equivalent to $K^{*}, g^{*}$ (see [2]). If $p$ is the quotient map of $N$ to $K^{*}$, standard arguments [2] show that $K^{*}, g^{*}$ generates the solenoid $\Sigma, h$ and $p$ induces a topological conjugacy of $\Lambda^{+}, f^{-1} / \Lambda^{+}$to $\Sigma, h$. By a similar argument, $\Lambda^{-}, f / \Lambda^{-}$ is topologically conjugate to $\Sigma^{t}, h^{t}$.

Claim 3. $\Omega(f) \supset \Lambda^{+} \cup \Lambda^{-}$. This follows immediately from Claim 2 .

Claim 4. $f$ is an Anosov-Smale diffeomorphism.

Axiom A, a. The hyperbolic splitting on $\Omega(f)$ is obtained as follows. $f^{-1}(f)$ is a contraction on the leaves of $\mathscr{G} N(\mathscr{G} M)$. By our construction also $f^{-1}(f)$ is expanding along $\Lambda^{+}\left(\Lambda^{-}\right)$, since $g$ and $g^{t}$ are expanding maps.

Axiom A, b. This is proved for any solenoid in [2].

Axiom B. Let $\delta N=T$, as before. As we noted in $\S 1$ there is a leaf $G$ of $\mathscr{G} N$ whose boundary represents a generator $\alpha_{j}$ of $H_{1}(T)$, if $G$ is on the $j$ th handle of $N$. Similarly, there is a leaf $G^{\prime}$ of $\mathscr{G} M$ whose boundary represents another generator $\beta_{j}$ of $H_{1}(T)$. It is clear that these leaves can be chosen so that their boundaries intersect, since each foliation of $N(M)$ extends to a foliation of $N \cup T(M \cup T)$. Q.E.D.

We note, but will not prove, that the "transpose" relationship established here between the source and the sink of $f$ is necessary as well as sufficient, i.e. no boarder class of pairs of solenoids can be realized as source and sink of Anosov-Smale diffeomorphism of $S^{3}$.

4. Properties of the diffeomorphisms. In this section we will prove an interesting fact about these diffeomorphisms, but first a more elementary fact.

Proposition 4.1. $f$ is not structurally stable. 
Proof. As noted above, the foliations $\mathscr{G} N$ and $\mathscr{G} M$, derived from the foliations of $N$ and $M$ by unstable and stable manifolds, respectively, can be extended to foliations of $T$. Choose a patch on $T$ where these foliations are as shown in Figure 9.

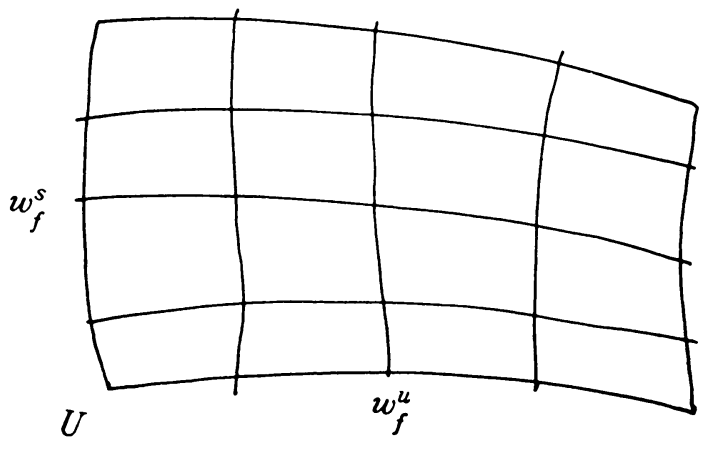

Figure 9

In a small neighborhood of the patch, perturb $f$ so that the foliations look like Figure 10. The foliation is not $C^{1}$ structurally stable, being the same phenomena described in [4]. Q.E.D.

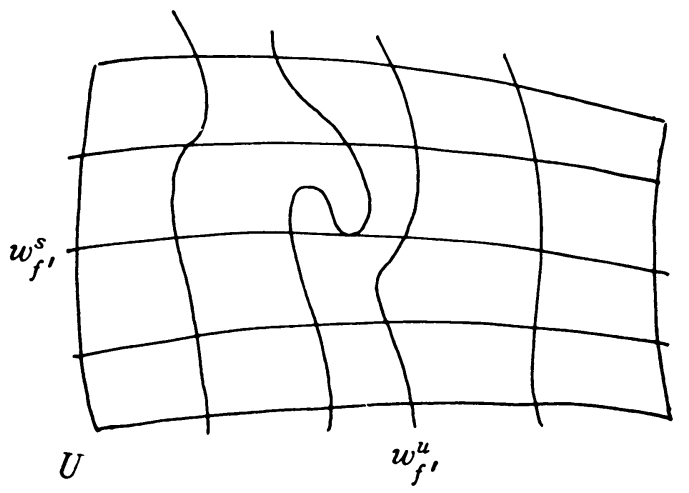

Figure 10

This method is adequate but somewhat strained as a way of constructing unstable points of tangency of stable and unstable manifolds of $f$. In fact, such points occur naturally in the diffeomorphisms we have constructed. We will treat only a special example, though the arguments clearly extend to all.

Let $f$ be a diffeomorphism whose source and sink are solenoids admitting the presentation: $K$ is a one-sphere and $g$ and $g^{t}$ are a double covering. This condition defines a class of diffeomorphisms, which we call $F_{2}$.

Proposition 4.2. There is a diffeomorphism, called $f_{2}$, of type $F_{2}$, which has the following property: there is a loop in $S^{3}$ such that at each point of the loop a stable 
manifold and an unstable manifold of $f_{2}$ are tangent. Furthermore, any sufficiently close $C^{1}$ approximation to $f_{2}$ also has this property.

Proof. Refer to a point at which a stable and an unstable manifold of $f_{2}$ are tangent as a tangency. Then the proposition says that $f_{2}$ has a stable loop of tangencies.

Let $K_{0}$ be the $z$-axis and $L_{0}$ be the unit circle in the $x$-y plane. $M$ is the "solid torus" having $L_{0}$ as its core, with internal radius one. $\mathscr{G} M$ is the family of flat vertical discs perpendicular to $L_{0}$. All this is shown in Figure 11. A leaf of $\mathscr{G} N$ is a

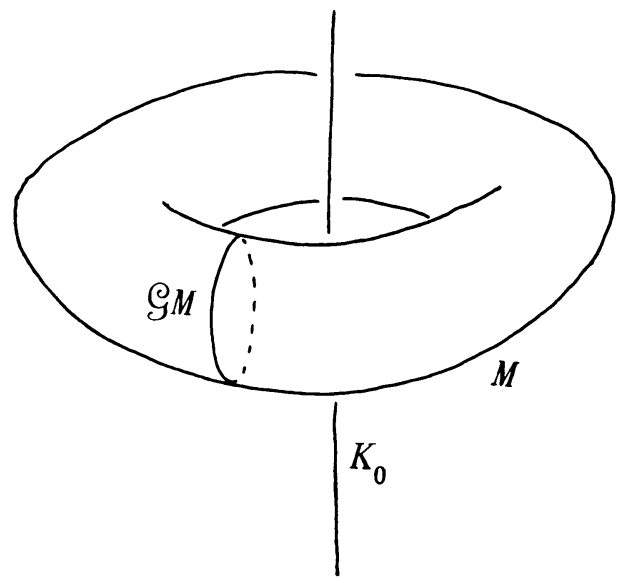

FigURE 11

"cap" which meets the boundary of $M$ on a circle. Let $G$ be such a leaf; $f_{2} / G$ is defined as follows. $f_{2}$ stretches $G$ and twists one end through $180^{\circ}$. Then $f_{2}$ folds the "loops" of $G$ together. In particular, $f_{2}$ twists $G$ in such a way that the result, viewed from any perspective, looks like Figure 12 . The vectors $u$ and $v$ are not

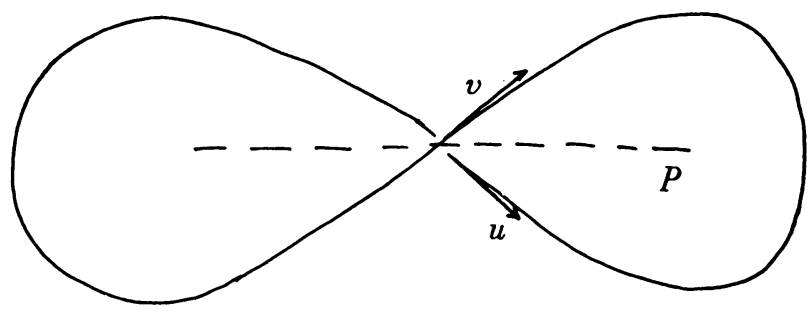

FIGURE 12

parallel, and there is a straight, "vertical" path in $G$ from the point of overpass to the point of underpass. Then the folding is done so as to leave a vertical plane $P$ (shown dotted) containing this path invariant. Lastly, embed this folded disc in $S^{3}$ so that its boundary runs around the interior of $M$ twice, the discs $f_{2}(G) \cap N$ are leaves of $\mathscr{G} N$, and the vertical plane is sent to a plane containing a leaf of $\mathscr{G} M$. 
That $f_{2}(G)$ is tangent to a leaf of $\mathscr{G} M$ follows from the fact that the folded disc is tangent to the vertical plane $P$. This is clearly an open condition since it requires only that the vectors $u$ and $v$ lie on opposite sides of $P$. By general position arguments we can arrange that the point of tangency be isolated.

Define a function $l: K_{0} \rightarrow S^{3}$ which assigns to each point $x$ of $K_{0}$ a point of tangency of $f_{2}\left(G_{x}\right)$ with a leaf of $\mathscr{G} M$, where $G_{x}$ is the leaf of $\mathscr{G} N$ through $x$. We must show that, for the proper choice of values if more than one is available, $l$ is continuous. To see this, take an interval $(a, b)$ about $x$ in $K_{0}$ and let $G_{(a, b)}$ be the union of the leaves of $\mathscr{G} N$ through $(a, b)$. This set is in the form of a solid cylinder, and $f_{2}$ acts to twist and fold it. Continuity follows from the openness of the condition defining a point of tangency. Q.E.D.

\section{BIBLIOGRAPHY}

1. S. Smale, Differential dynamical systems, Bull. Amer. Math. Soc. 73 (1967), 747-817. MR 37 \#3598.

2. R. Williams, One-dimensional non-wandering sets, Topology 6 (1967), 473-487. MR 36 \#897.

3. L. Neuwirth, Knot groups, Ann of Math. Studies, no. 56, Princeton Univ. Press, Princeton, N. J., 1965. MR 31 \#734.

4. S. Smale, Structurally stable systems are not dense, Amer. J. Math. 88 (1966), 491-496. MR 33 \#4911.

Department of Mathematics, Northwestern University, Evanston, Illinois 60201

Current address: Chicago State University, Chicago, Illinois 60621 